\title{
Metabolic Outcomes of Surgery for Non-Obese Type 2 Diabetes
}

\author{
Kyung Yul Hur, M.D., Ph.D. \\ Department of Surgery, Soonchunhyang University Seoul Hospital, Soonchunhyang University College of Medicine, Seoul, Korea
}

\begin{abstract}
The most plausible contributing factor to non-obese type 2 diabetes may be imbalanced incretin release from the intestinal epithelium in response to nutrients. Rerouting intestinal continuity through bypass surgery to modulate incretin release is therefore a reasonable treatment. We believe that a major determinant of metabolic outcomes is entire duodenal exclusion without leaving any duodenal epithelium and exclusion of sufficient length of jejunum. More importantly, the procedure should be implemented with safety and without sequelae. To achieve this, we invented a novel procedure with acceptable surgical safety and excellent and durable metabolic outcomes. Post-surgical intestinal adaptation should be considered to achieve successful outcomes.
\end{abstract}

Keywords: Type 2 diabetes, Metabolic surgery, Physiologic adaptation

\author{
Received March 20, 2020 \\ Revised May 22, 2020 \\ Accepted May 26, 2020 \\ Corresponding author \\ Kyung Yul Hur \\ Department of Surgery, \\ Soonchunhyang University Hospital, \\ 59 Daesagwan-ro, Yongsan-gu, \\ Seoul 04401, Korea \\ Tel: $+82-2-709-9114$ \\ Fax: +82-2-709-9246 \\ E-mail: hurusa@hanmail.net \\ ORCID: \\ https://orcid.org/0000-0003-0560-6434
}

Copyright ( 2020 The Journal of Minimally Invasive Surgery. All rights reserved.

\section{INTRODUCTION}

In contrast to type 2 diabetes with obesity, multiple factors are usually involved in type 2 diabetes without obesity. Among them, the most plausible contributing factor is imbalanced incretin release from the intestinal epithelium in response to nutrients. However, the precise mechanisms underlying the surgical improvement of diabetes remain unclear and appear to be complex. Furthermore, possible complications from surgery remain a point of concern. Diabetes, an inability to process blood glucose, is regarded as progressive and incurable. Therefore, the best nonsurgical treatment option is delaying the progression of the disease and minimizing diabetic complications. In contrast, the aim of surgical treatment should be enabling blood-glucose processing without the assistance of insulin or oral hypoglycemic agents. Outcomes of metabolic surgery are usually extreme. Diabetes improvement is considered to indicate successful surgery, whereas exacerbation or stagnation is indicative of a failed operation. The lack of a requirement for medical treatments after surgery should be considered an acceptable outcome. Decreased serum concentrations of glycated hemoglobin, which reflects blood-glucose processing, is also acknowledged as an acceptable outcome. Individuals whose outcomes are unacceptable or unfavorable have a pattern of recurrent hyperglycemia with increased concentrations of glycated hemoglobin within first year after surgery, and frequently within 3 6 months postoperatively. Therefore, the absence of recurrent hyperglycemia first year after surgery is a promising sign of operative success and suggests an acceptable outcome thereafter. The assessment of the outcomes of the surgery is extreme. Researcher is whether one of advocator ${ }^{1}$ or criticizer. ${ }^{2}$ Intriguingly, metabolic outcomes 
of duodenal-jejunal bypass (DJB) surgery which is most commonly performed metabolic surgery for non-obese type 2 diabetes are inconsistent.

Interestingly, a simple modified surgical technique has been applied differently in individuals with unsuccessful surgery. During the DJB procedure, the shortcut jejunal end (upper pole of Roux limb) is connected to either the pyloric portion of the stomach or the 1st portion of the duodenum just distal to the sphincter. The name of applied surgical technique has been described in the related articles as gastrojejunostomy or duodenojejunostomy, respectively. Curiously, most unfavorable outcomes are associated with duodenojejunostomy, which is end procedure of pylorus preserving technique to assure comfortable digestive function after surgery. However, pyloric sphincter muscle and duodenum is adjoined together, total duodenal exclusion without leaving small amount of duodenal epithelium concomitantly, is known to impossible practically in pylorus function preservation technique, so far. Finally, successful surgery also depends on the length of the bypassing segment. For modulation of incretin release from the epithelium in response to nutrients, the location of incretin secreting enteroendocrine cells is very important. ${ }^{3}$

We herein investigated the possible pathophysiological relationships among pylorus preservation, remnant duodenal epithelium, the time to recurrence after surgery, and the unique patterns of epithelial regeneration and enteroendocrine cell distribution.

\section{PROPOSED MECHANISM OF BLOOD GLUCOSE CONTROL AND RECURRENCE}

For successful metabolic surgery, a comprehensive understanding of all possible relevant physiology associated with resolution or recurrence of diabetes is crucial. These understanding must include blood glucose regulation with physiologic divergence in glucose dependent insulinotropic polypeptide (GIP), which is released from the proximal small intestinal epithelium in response to nutrients and is considered as a causal element in type 2 diabetes. ${ }^{4}$ Other important factors are the intricately organized cell regeneration of the intestinal epithelium, the maintenance of the integrity of the intestinal epithelial lineage and the pattern of gastrointestinal adaptation after small bowel bypass, which is similar to the adaptive response after massive small bowel resection. ${ }^{5,6} \mathrm{Fi}^{-}$ nally, the locations of enteroendocrine cells must be identified.

\section{GIP}

Nutrients stimulate insulin secretion either directly or through incretin hormones. Glucagon like peptide-1 (GLP-1) has received considerably more research attention than other incretin peptides because of its clinical effectiveness in the treatment of type 2 diabetes. In contrast, GIP is considered as ineffective peptide that does not stimulate insulin secretion in people with type 2 diabetes. However, recent studies have suggested that GIP has a physiologic function in blood glucose stabilization. GIP appears to regulate glucose levels through the modulation of two pancreatic counter-regulatory hormones?

Patients with type 2 diabetes experience fasting and postprandial hyperglucagonemia, which contribute to the hyperglycemia characterizing these patients. Clinical studies have shown that the gastrointestinal hormone GIP might play an important role in this pathophysiological phenomenon. Furthermore, potentially effective treatment strategies for patients with type 2 diabetes include suppression of glucagon secretion or antagonization of glucagon receptors. Therefore, metabolic surgery should aim to reduce serum GIP levels after meals, thereby modulating the postprandial glucose surge. In addition, recent reports support the role of postprandial glucose control as an important strategy to consider in comprehensive management plans for individuals with diabetes. ${ }^{8}$ Conclusively, the aim of metabolic surgery in patients without obesity should be to reduce GIP release after meals while effectively increasing GLP-1 without deterioration of digestive quality or function.

\section{Intestinal epithelial regeneration and maintenance of cell lineage}

Human intestinal epithelial regeneration occurs within the crypt-villus axis. Stem cells are located at the bases of the crypts of Lieberkühn. From the bottom of the crypt, the progenies of these dividing cells migrate upward to the villi surfaces and are finally sloughed from the tips of the villi. The architectural modeling and cellular identity of the intestinal epithelium depend on epithelial-mesenchymal interactions during migration. ${ }^{5}$ Thus, the mesenchymal layer just below the epithelium has a key role in maintaining the epithelial lineage.

\section{Compensatory proliferation as a physiologic adaptation}

Compensatory hypertrophy and proliferation of residual small intestine after bypass surgery is a normal physiologic response to nutritional insufficiency. ${ }^{9}$ The compensatory response is rapid at the distal intestine from anastomosis. Reconstruction with rearrangement of the intestinal continuity followed by shuffling of apposing villi and crypts with displacement of the epithelial layer and counterpart mesen- 
chymal layer results in scattering of the epithelial lineage at both ends of the intestine. The intestinal mucosal surface is crowded with villi and crypts. Each villus is surrounded by approximately 15 crypts containing 350 400 cells. ${ }^{10}$ After duodenojejunostomy, along with a strong compensatory proliferative reaction in the distal intestine, rapid proliferation of the displaced duodenal epithelial lineage to the opposite area results in tissues more like the duodenum than the jejunum. This process has been called as regional reprogramming to mature proximal identity and transcriptional proximalization. ${ }^{11}$ Until sufficient cell mass growing for recognizable recurrent hyperglycemia takes several months, thus possibly explaining the delay before recurrent hyperglycemia is observed. Remarkably, in short bowel syndrome, the adaptation of the small intestine lasts for up to 1 year, but the maximum proliferation occurs until 6 months. ${ }^{12}$

\section{Transcriptional proximalization}

Evidence of epithelial lineage migration has recently been described. A single cell analysis has revealed regional reprogramming during adaptation to massive small bowel resection in mice. ${ }^{11}$ According to that article, the change from distal intestinal epithelium to a proximal identity after proximal small bowel resection is accompanied by increased proximal small intestinal nutrient processing. Another evidence is surgical transposition of a segment of the ileum to a more proximal jejunum has been reported to result in structural and functional jejunalization of the transposed ileum both morphologic and histologically in canine experiment. ${ }^{13}$ Intriguingly, different outcomes have been observed between surgical pylorus preserving DJB and duodenal-jejunal bypass liner (DJBL). The DJBL is a flexible sleeve that is endoscopically implanted within the small intestine. ${ }^{14}$ Despite bypassing almost the same area as pylorus-preserving surgical DJB, DJBL rarely results in recurrent hyperglycemia while the barrier is in place. The barrier separates small intestinal epithelium with ingested food $60 \mathrm{~cm}$ from the 1st portion of the duodenum. ${ }^{14}$ Patients who undergo surgical pylorus-preserving DJB have a pattern of recurrent hyperglycemia from 3 to 6 months after operation. In contrast, patients treated with a DJBL rarely show recurrence while the barrier is in place. The long distance between the uncovered intestinal epithelium may prevent epithelial lineage migration, thus resulting in better metabolic outcomes than those of surgical DJB. The most effective metabolic surgery for patients with low-BMI type 2 diabetes is classic biliopancreatic diversion (BPD). Outcomes of the modified form of BPD with duodenal switch are not as favorable as those of classic BPD. ${ }^{15}$

\section{Where and how to bypass}

The edge of the proximal bowel epithelium that is continuously connected to the cut end of the jejunum (Roux limb) appears to strongly affect post-operative recurrent hyperglycemia. In the DJB procedure, the possible area of the edge of the proximal bowel is either the pyloric portion of the stomach or the first portion of the duodenum. Both procedures have pros and cons. Pyloric sphincter preservation is important to maintain postoperative digestive function. To preserve the pyloric sphincter muscle completely, the adjoined duodenal epithelium should be preserved. Total exclusion of the duodenum without leaving any duodenal epithelium in the pylorus preserving procedure has been considered practically impossible to date.

The total length of the biliopancreatic (BP) limb also strongly affects metabolic outcomes, regardless of the length of the alimentary limb. Duodenum is always totally excluded in Roux-en-Y gastric bypass, single anastomosis gastric bypass and classic biliopancreatic diversion. Therefore, the only determinant of metabolic outcomes is BP limb length. Thus, confirming the relationships between the length of the BP limb and postoperative GIP changes compare to preoperative value would be possible. Post-operative GIP changes may be negatively correlated with BP limb length. ${ }^{16-20}$ However, metabolic outcomes and the length of the BP limb appear to be positively correlated (Table 1). Postoperative GIP changes and

Table 1. Relationship between BP limb length and GIP alteration with metabolic outcomes

\begin{tabular}{|lccccc|}
\hline \multicolumn{1}{|c}{ Reference } & Operation & Alimentary limb $(\mathrm{cm})$ & BP limb $(\mathrm{cm})$ & GIP alteration & Metabolic outcomes \\
\hline Laferrère et al. $^{16}$ & RnY & $150 \mathrm{~cm}$ & $30 \mathrm{~cm}$ & $105 \%$ & Mild $^{*}$ \\
\hline Laferrère et al. $^{17}$ & RnY & $150 \mathrm{~cm}$ & $40 \mathrm{~cm}$ & $103 \%$ & Mild $^{*}$ \\
\hline Fellici et al. $^{18}$ & RnY & $150 \mathrm{~cm}$ & $100 \mathrm{~cm}$ & $90 \%$ & Moderate $^{*}$ \\
\hline Kim et al. $^{20}$ & SAGBP & $0 \mathrm{~cm}$ & $200 \mathrm{~cm}$ & $53 \%$ & Good $^{*}$ \\
\hline Guidone et al. $^{19}$ & BPD & $200 \mathrm{~cm}$ & $250 \mathrm{~cm} \uparrow$ & $49 \%$ & Excellent $^{*}$ \\
\hline
\end{tabular}

$\mathrm{RnY}=$ Roux-en-Y gastric bypass; SAGBP = single anastomosis gastric bypass; BPD = biliopancreatic diversion; BP limb = biliopancreatic limb; GIP = glucose dependent insulinotropic polypeptide. *Author's view based on conclusion of each article. 
the length of the BP limb are inversely proportional. At a BP limb length of approximately $100 \mathrm{~cm}$, the GIP level is almost the same before and after surgery. A longer BP limb length results in a lower post-operative than pre-operative GIP level, whereas a shorter BP limb results in a higher GIP level postoperatively than preoperatively. ${ }^{16-20}$ In classic BPD, the longest BP limb results in excellent outcomes for type 2 diabetes, with a change in GIP to less than half its preoperative value. In contrast, in patients with a short BP limb, the GIP is more than 100 percent its preoperative value. These findings provide solid evidence of the important role of the BP limb and indicate that decreasing GIP is a key factor in curing diabetes.

With respect to the efficacy of nutritional absorption, looptype reconstruction is better than the Roux-en-Y pattern. Depending on the purpose of the operation, the expected function of the Roux limb varies. Prevention of nutrient absorption is desirable in bariatric surgery, whereas reducing enteric hormone release is desirable in metabolic surgery. Without digestive enzymes, nutritional absorption is interrupted, however, the lack of digestive enzymes does not interfere with incretin secretion. Unnecessary perturbation of nutrient absorption is another disadvantage of Roux-en-Y gastric bypass surgery for non-obese individuals. According to a recent study comparing the two procedures in the treatment of non-obese type 2 diabetes, single-anastomosis gastric bypass has been found to achieve better glycemic control than Roux-en-Y gastric bypass. A possible mechanism for this advantage in diabetic management is the much longer BP limb in single-anastomosis gastric bypass. ${ }^{21}$ In patients undergoing metabolic surgery, especially low BMI subjects, loop-type reconstruction of gut integrity has advantages over Roux-en-Y bypass.

The recommended area of foregut bypass is $200 \mathrm{~cm}$ distal to the ligament of Treitz. Exclusion of the duodenum and the proximal $200 \mathrm{~cm}$ from the duodenal jejunal junction of the je- junum ensures an adequate area for nutrient absorption while decreasing the $\mathrm{K}$-cell population to minimize GIP release and increasing the population of L cells to maximize GLP-1 production. ${ }^{22,23}$

\section{THREE PRINCIPLES FOR METABOLIC SURGERY IN NON-OBESE PATIENTS WITH TYPE 2 DIABETES}

Safety is essential in the surgical treatment of type 2 dia- $^{-}$ betes. Metabolic outcomes should be judged in conjunction with safety outcomes. Permanent metabolic effect is as important as safety. Therefore, metabolic surgery should focus on safety, effectiveness and durability. However, meeting all these prerequisites is practically challenging. To overcome these obstacles, three principles are necessary: 1) preservation of pyloric sphincter function, 2) total duodenal exclusion and 3) creation of a BP limb of at least $200 \mathrm{~cm}$. Placement of interrupted sutures between the pyloric muscle and the small intestine is very important to preserve pyloric sphincter function and avoid restriction of pyloric sphincter movement. Duodenal epithelium attached to the distal end of the pyloric sphincter should be removed. Excision or cauterization should be used to devitalize duodenal tissue. The transitional zone between the duodenum and stomach can easily be discriminated with the naked eye. Therefore, anastomosis between the pyloric sphincter and the small intestine should be conducted under direct vision. The abdominal wall is very thin in non-obese compared with obese patients. Therefore, a skin incision less than $5 \mathrm{~cm}$ is sufficient to perform extracorporeal anastomosis. A BP limb of $200 \mathrm{~cm}$ is adequate to maximize antidiabetic effects. The longer the biliopancreatic limb, the better the surgical outcomes. However, because of the possibility of malabsorption, a BP limb longer than $200 \mathrm{~cm}$ is not recommended.

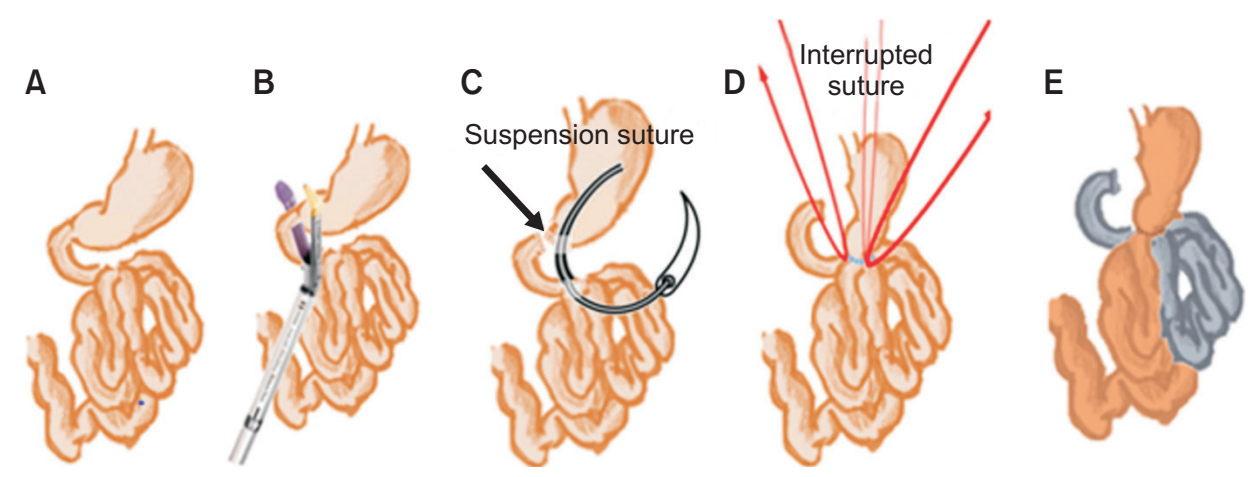

Fig. 1. Single anastomosis duodenal-jejunal bypass with total exclusion of duodenum in the sequence. (A) Preoperative state. (B) Frist portion of duodenum just distal to pyloric sphincter is transected with linear stapler. (C) Suspension sutures are made between distal end of stomach and $200 \mathrm{~cm}$ distal from Treitz ligament of jejunum. (D) Anastomosis is conducted between the pyloric sphincter muscle and the small intestine under the direct vision. (E) The single anastomosis duodenal-jejunal bypass with total exclusion of duodenum is completed. 
No surgical procedure has satisfied all three goals stated above to date. However, the second and third goals, which are associated with antidiabetic effectiveness, can be achieved with classic BPD, Roux-en-Y and mini-gastric bypass with more than $200 \mathrm{~cm}$ of BP limb. 24,25

\section{SATISFYING THE THREE PRINCIPLES SIMULTANEOUSLY}

We have been conducting single-anastomosis DJB with total exclusion of duodenum since 2015. Anastomosis between small intestine with pyloric sphincter muscle directly, satisfies all three principles described above. The safety and feasibility of anastomosis between the pyloric sphincter muscle and small intestine have already been confirmed in other surgical procedures. There had been no serious complications in a series of more than 100 consecutive pylorus preserving pancreaticoduodenectomies which conducted by the author. Although our data are not published yet, we have conducted with 78 subjects of non-obese type 2 diabetes, whose BMI less than $30 \mathrm{~kg} / \mathrm{m}^{2}$ (Fig. 1). The mean BMI was $24.8 \mathrm{~kg} / \mathrm{m}^{2}$, and the lowest BMI in our series was $18 \mathrm{~kg} / \mathrm{m}^{2}$. The surgical outcomes of the 78 patients who have undergone operation have been acceptable without any complications, and most patients have been satisfied with their outcomes. The procedure is very simple and easy to perform, and it requires a short operative time. Interestingly, lower BMIs are associated with better outcomes. The surgery is not recommended for BMI above $30 \mathrm{~kg} / \mathrm{m}^{2}$, because it would be scarcely effective, owing to the predominant deleterious effect of lipid.

\section{CONCLUSION}

Metabolic surgery to treat non-obese type 2 diabetes is challenging. Surgical safety without sequelae is more important than metabolic outcomes. To meet the desired goals, three principles are recommended. Metabolic improvement after surgery appears to depend on the post-operative versus preoperative GIP value. The length of the biliopancreatic limb strongly affects the overall antidiabetic outcomes, and recurrent hyperglycemia, the main cause of disappointing outcomes, appears to be related to not excluded duodenal epithelium.

\section{ORCID}

Kyung Yul Hur, https://orcid.org/0000-0003-0560-6434

\section{CONFLICT OF INTEREST}

None.

\section{FUNDING}

This work was supported in part by the Soonchunhyang University Research Fund.

\section{ACKNOWLEDGMENTS}

I would like to thank prof. Sang Hyun Kim working with me as a new colleague, and prof. Yong Jin Kim who helped me come up with these days too. I also express my deep gratitude and respect to the Ha Eun Kim coordinator and a nurse who assisted me at outpatient clinic, Sun Joo Song.

\section{REFERENCES}

1) Rubino F, Marescaux J. Effect of duodenal-jejunal exclusion in a non-obese animal model of type 2 diabetes: a new perspective for an old disease. Ann Surg 2004;239:1-11.

2) Klein S, Fabbrini E, Patterson BW, et al. Moderate effect of duodenal-jejunal bypass surgery on glucose homeostasis in patients with type 2 diabetes. Obesity (Silver Spring) 2012;20:1266-1272.

3) Polak JM, Bloom SR, Kuzio M, Brown JC, Pearse AG. Cellular localization of gastric inhibitory polypeptide in the duodenum and jejunum. Gut 1973;14:284-288.

4) Lund A, Bagger JI, Christensen M, Knop FK, Vilsb øll T. Glucagon and type 2 diabetes: the return of the alpha cell. Curr Diab Rep 2014;14:555.

5) Crosnier C, Stamataki D, Lewis J. Organizing cell renewal in the intestine: stem cells, signals and combinatorial control. Nat Rev Genet 2006; 7:349-359.

6) Warner BW. The Pathogenesis of Resection-Associated Intestinal Adaptation. Cell Mol Gastroenterol Hepatol 2016;2:429-438.

7) Christensen M, Vedtofte L, Holst JJ, Vilsb øll T, Knop FK. Glucose-dependent insulinotropic polypeptide: a bifunctional glucose-dependent regulator of glucagon and insulin secretion in humans. Diabetes 2011;60:3103-3109.

8) Madsbad S. Impact of postprandial glucose control on diabetesrelated complications: How is the evidence evolving? J Diabetes Complications 2016;30:374-385.

9) Dudrick SJ, Daly JM, Castro G, Akhtar M. Gastrointestinal adaptation following small bowel bypass for obesity. Ann Surg 1977; 185:642-648.

10) Dalal J, Radhi M. Intestinal Stem Cells: Common Signal Pathways, Human Disease Correlation, and Implications for Therapies. ISRN Stem Cells 2013;2013:372068.

11) Seiler KM, Waye SE, Kong W, et al. Single-Cell Analysis Reveals 
Regional Reprogramming During Adaptation to Massive Small Bowel Resection in Mice. Cell Mol Gastroenterol Hepatol 2019;8: 407-426.

12) Sukhotnik I. Short bowel syndrome: intestinal adaptation. In: Rintala RJ, Pakarinen M, Wester T, editors. Current Concepts of Intestinal Failure. Cham: Springer International Publishing; 2016. p.23-37.

13) Smithy WB, Cuadros CL, Johnson H, Kral JG. Effects of ileal interposition on body weight and intestinal morphology in dogs. Int J Obes 1986;10:453-460.

14) Jirapinyo P, Haas AV, Thompson CC. Effect of the DuodenalJejunal Bypass Liner on Glycemic Control in Patients With Type 2 Diabetes With Obesity: A Meta-analysis With Secondary Analysis on Weight Loss and Hormonal Changes. Diabetes Care 2018;41: 1106-1115.

15) Cossu ML, Noya G, Tonolo GC, et al. Duodenal switch without gastric resection: results and observations after 6 years. Obes Surg 2004:14:1354-1359.

16) Laferrère B, Teixeira J, McGinty J, et al. Effect of weight loss by gastric bypass surgery versus hypocaloric diet on glucose and incretin levels in patients with type 2 diabetes. J Clin Endocrinol Metab 2008;93:2479-2485.

17) Laferrère $B$, Heshka $S$, Wang $K$, et al. Incretin levels and effect are markedly enhanced 1 month after Roux-en-Y gastric bypass surgery in obese patients with type 2 diabetes. Diabetes Care 2007;30: 1709-1716.

18) Fellici AC, Lambert G, Lima MM, et al. Surgical treatment of type
2 diabetes in subjects with mild obesity: mechanisms underlying metabolic improvements. Obes Surg 2015;25:36-44.

19) Guidone C, Manco M, Valera-Mora E, et al. Mechanisms of recovery from type 2 diabetes after malabsorptive bariatric surgery. Diabetes 2006;55:2025-2031.

20) Kim MJ, Park HK, Byun DW, Suh KI, Hur KY. Incretin levels 1 month after laparoscopic single anastomosis gastric bypass surgery in non-morbid obese type 2 diabetes patients. Asian J Surg 2014; 37:130-137.

21) Almalki OM, Lee WJ, Chong K, Ser KH, Lee YC, Chen SC. Laparoscopic gastric bypass for the treatment of type 2 diabetes: a comparison of Roux-en-Y versus single anastomosis gastric bypass. Surg Obes Relat Dis 2018;14:509-515.

22) Mortensen K, Christensen LL, Holst JJ, Orskov C. GLP-1 and GIP are colocalized in a subset of endocrine cells in the small intestine. Regul Pept 2003;114:189-196.

23) Guedes TP, Martins S, Costa M, et al. Detailed characterization of incretin cell distribution along the human small intestine. Surg Obes Relat Dis 2015;11:1323-1331.

24) Mingrone $G$. Role of the incretin system in the remission of type 2 diabetes following bariatric surgery. Nutr Metab Cardiovasc Dis 2008;18:574-579.

25) Kim MJ, Hur KY. Short-term outcomes of laparoscopic single anastomosis gastric bypass (LSAGB) for the treatment of type 2 diabetes in lower BMI $(<30 \mathrm{~kg} / \mathrm{m}(2))$ patients. Obes Surg 2014;24: 1044-1051. 